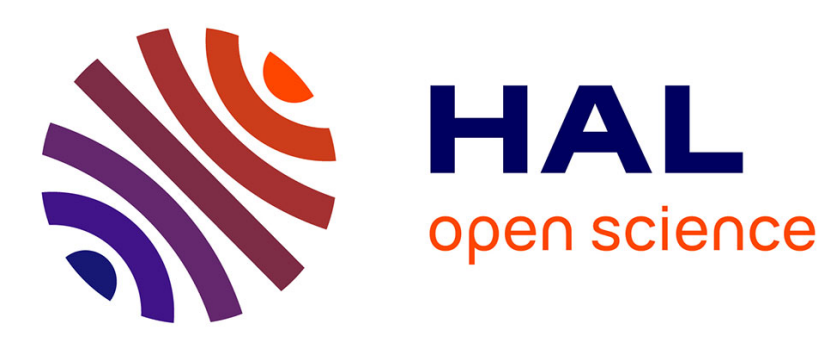

\title{
Relaxation of the Off-Centered H- ion at E'4 Defect Center in $\alpha$-Quartz
}

\author{
A. Manov, M. Kirtcheva, L. Mihailov
}

\section{To cite this version:}

A. Manov, M. Kirtcheva, L. Mihailov. Relaxation of the Off-Centered H- ion at E'4 Defect Center in $\alpha$-Quartz. Journal de Physique IV Proceedings, 1996, 06 (C8), pp.C8-531-C8-534. 10.1051/jp4:19968115 . jpa-00254545

\section{HAL Id: jpa-00254545 https://hal.science/jpa-00254545}

Submitted on 1 Jan 1996

HAL is a multi-disciplinary open access archive for the deposit and dissemination of scientific research documents, whether they are published or not. The documents may come from teaching and research institutions in France or abroad, or from public or private research centers.
L'archive ouverte pluridisciplinaire HAL, est destinée au dépôt et à la diffusion de documents scientifiques de niveau recherche, publiés ou non, émanant des établissements d'enseignement et de recherche français ou étrangers, des laboratoires publics ou privés. 


\title{
Relaxation of the Off-Centered $\mathrm{H}^{-}$ion at $\mathbf{E}_{4}^{\prime}$ Defect Center in $\alpha$-Quartz
}

\author{
A. Manov, M. Kirtcheva and L. Mihailov \\ Institute of Solid State Physics, Bulgarian Academy of Sciences, 72 Tzarigradsko Chaussee, 1784 Sofia, \\ Bulgaria
}

\begin{abstract}
The cluster calculations of the configuration with energy minima for $\mathbf{E}_{4}{ }_{4}$ defect center indicate a doublewell potential energy profile for off-centered displacement of the $\mathrm{H}^{-}$ion substituting for the oxygen at the $\mathrm{E}_{4}{ }_{4}$ center in $\alpha$-quartz. Pseudo Jahn-Teller effect formalism is applied to interpret the cluster calculated data. The Schrödinger equation with pseudo Jahn-Teller potential energy is numerically solved. The eigenvalue spectrum and corresponding wave functions of these lowered symmetry centers are found. Applying a modified reaction rate method the relaxation times of $\mathrm{H}^{-}$ion are computed. The acoustic losses $Q^{-1}$ are computed and compared to experimental data. The specific profile of the adiabatic potential energy, the calculated energy spectrum and the corresponding relaxation times denote that $\mathrm{H}^{-}$ion is a mixed tunneling-relaxation system. The presented theoretical results may be useful in forecasting the radiation response of $\alpha$-quartz.
\end{abstract}

\section{INTRODUCTION}

In our preceding communications the $\mathrm{H}^{-}$ion at $\mathrm{E}_{4}^{\prime}$ defect centers in $\alpha$-quartz was interpreted in terms of a pseudo Jahn-Teller effect (PJTE) [1] and the two-well adiabatic potential energy profile was estimated by fitting procedure with Isoya cluster data calculation [2]. The reaction rate method was applied to calculate the temperature dependence of the relaxation times [1]. The corresponding transitions along the potential energy profile are temperature-independent in one direction, while in the reverse direction are temperature dependent. The reason is that the lowest level of the system falls in the deeper well of the obtained PJT potential (Fig. 1a). Taking into account this dependence and assuming that $\mathrm{H}^{-}$ion relaxes classically, the acoustic losses and frequency offset for $5 \mathrm{MHz}$ AT-cut quartz resonator were calculated by the Debye formula [3]. These theoretically calculated acoustic losses were not compared with appropriate experimental data.

The experimental data show that there exist a peak in dielectric losses at $5 \mathrm{kHz}$ in X-irradiated $\alpha$ quartz at $26 \mathrm{~K}$ and in fast-neutron-irradiated $\alpha$-quartz at $\sim 32 \mathrm{~K}$ [4]. Following Weeks \& Nelson [5], the most of $E_{2}^{\prime}-E^{\prime}{ }_{4}$ defect centers are formed after $X$-ray- and $\gamma$-ray-irradiation. For this reason, one may expect the $\mathrm{H}^{-}$ion relaxation peak at $\sim 26 \mathrm{~K}$. According to the aforementioned calculation [3], the relaxation peak in the acoustic losses should be appeared near $36 \mathrm{~K}$ at $5 \mathrm{kHz}$ external field frequency.

A criterion for the applicability of the model for description of $\mathrm{H}$ ion and the method for calculation of its relaxation behaviour is the comparison with the experimental data. Moreover, the special type of the adiabatic potential energy profile (see Fig. 1) and of the temperature dependencies of the relaxation times had been provided a peculiar system (not pure Debye - type) for acoustic losses calculation. Thus, a more careful approach is necessary to apply in description of the relaxation of the $\mathbf{H}$ ion and in calculation of the related acoustic losses. The aims of this paper are to describe more precisely the PJT energy profile, to compute numerically the energy spectrum, to calculate the corresponding relaxation times and the acoustic losses which have to be compared with experimental data. 


\section{PJTE POTENTIAL ENERGY PROFILE DESCRIPTION AND ENERGY SPECTRUM CALCULATION}

The following essential improvements of the off-centered $\mathrm{H}^{-}$ion at $\mathrm{E}_{4}^{\prime}$ defect center in $\alpha$-quartz relaxation description are proposed: (1) A better PJTE fit to the Isoya's cluster data; (2) The Schrödinger equation solves numerically in order to compute the energy spectrum; (3) Application of the improved adapted reaction-rate method [6] which includes: (i). Numerical calculation of quasiclassical transition probabilities; (ii). Calculation of the transition probabilities for the lowest levels according to the tunneling model (TM); (iii). Taking account of the occupations of all energy levels of the system.

The fit to the Isoya's cluster data [2] using PJT potential with asymmetry energy $\Delta=0.105 \mathrm{eV}$ [1] is not the best one. If the coordinate of the interwell maximum is a fitting parameter, then one may obtain a better fit with $\Delta=0.063 \mathrm{eV}$. For this purpose a multidimensional optimization method is applied. This procedure is realized by combined random searching (complex method) and gradient method (Davidon-Fletcher-Powel). The obtained PJT parameters $\left(E_{g u}=7.4 \mathrm{eV}, G=11.99 \mathrm{eV} / \AA, \mathrm{K}=29.65 \mathrm{eV} / \AA^{2}\right.$; for comparison see PJT parameters [1]) considerably change the system properties and the $\mathrm{H}$ ion turns from a system similar to classical relaxation system (RS) into a system with eventual tunneling properties.

The computed energy spectrum shows that in the first case $(\Delta=0.105 \mathrm{eV})$ there exist one single level in the deeper well and large difference between the following levels (Fig. 1a), while in the next case (the better fit with $\Delta=0.063 \mathrm{eV}$ ) the lowest two energy levels are common for the both wells and the possibility for tunneling between them becomes essential (Fig. 1b).

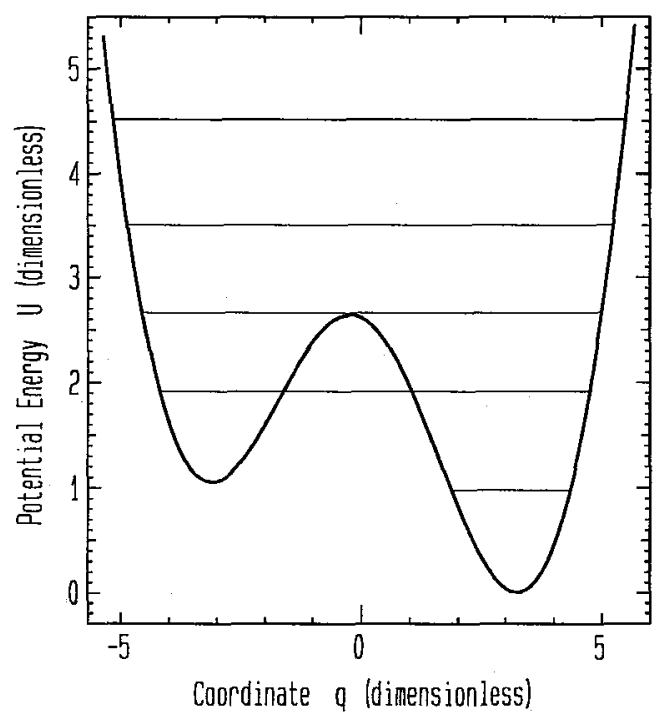

(a)

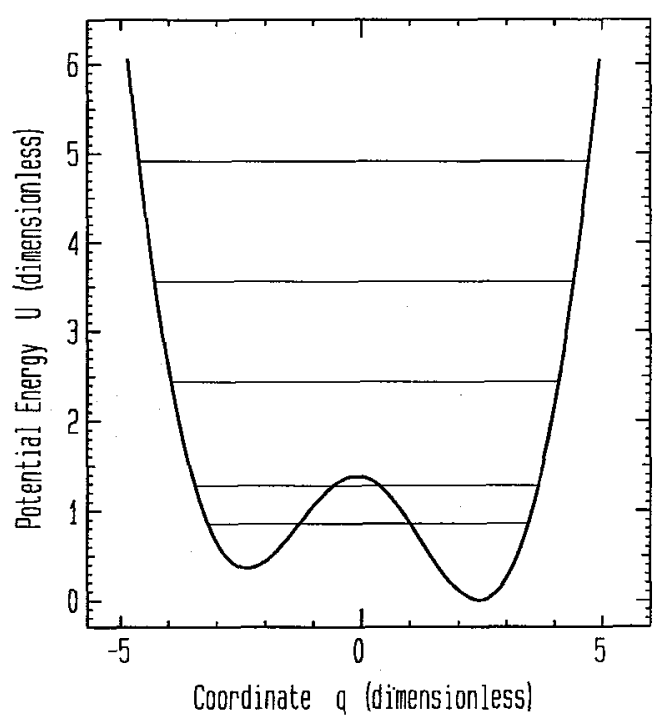

(b)

Figure 1. Dimensionless double well potential energies U(q) with numerically calculated vibronic levels of $\mathrm{H}^{-}$ion at $\mathrm{E}_{4}{ }_{4}$ center in $\alpha$-quartz.

\section{HI. RELAXATION TIMES AND ACOUSTIC LOSSES}

Interwell transfer rate (relaxation rate) can be calculated on the base of the potential energy profile and the energy levels by the adapted for non-radiative transitions Reaction-Rate Method (RRM) [7]. In accordance with this method, $\mathrm{H}^{-}$ion relax via tunneling in ground state $(T=0)$, via thermally activated tun- 
neling in remaining subbarrier states, and via classical transitions in overbarrier states [1]. In the present calculations an improved RRM is applied [6] to calculate the temperature dependence of the relaxation rates on the base of the numerically computed energy levels. The relaxation rate for the lowest couple of tunnel splitted energy levels is calculated in accordance with the standard TM. A corresponding rates for the remaining (sub- and overbarrier) energy levels are numerically computed using quasiclassical approximation.

As it is explained in [6], Tunneling-Relaxation System (TRS) may relax as a tunneling system (TS) at low temperature, or classically as a relaxation system (RS) at high temperature. At some characteristic temperature $T_{c}$ the TS and RS relaxation times are equal. In order to calculate the relaxation losses in accordance with both TM and Debye relaxation ratio, "tunneling degree" parameter $\lambda$ (the ratio between the tunneling transition probability and the total one) and "separation degree" $\mu$ (the thermal occupation probability of the lowest couple tunneling levels) are introduced. The relaxation strength is constructed as a sum of two terms with weight coefficients $\zeta$ and $1-\zeta$ (accounting for TS and RS contribution correspondingly). $\zeta$ is defined as $\zeta(T)=\lambda(T) \mu(T)$.

The application of that new method for the relaxation times and losses calculation upon the case with $\Delta=0.105 \mathrm{eV}$ leads to the shift of the $\mathrm{H}^{-}$ion absorption peak $T_{0}$ from $\sim 56 \mathrm{~K}$ [3] to $\sim 130 \mathrm{~K} \mathrm{[6]}$ at frequency of the external field $f=5 \mathrm{MHz}$. In this case the sound absorption peak temperature $T_{0}$ is very near to the characteristic temperature $T_{c}$ (see Fig. 2a) at which this off-position center is proved as tunneling and relaxation system simultaneously. The result clearly shows the mixed tunneling-relaxation character of the system. In the case $\Delta=0.063 \mathrm{eV}$ the peak appears at $T_{0} \sim 38 \mathrm{~K}$ at $f=5 \mathrm{MHz}$ and $T_{0} \ll T_{c}$ (see Fig. $2 \mathrm{~b}$ ). This means that the character of the system is very similar to TS.

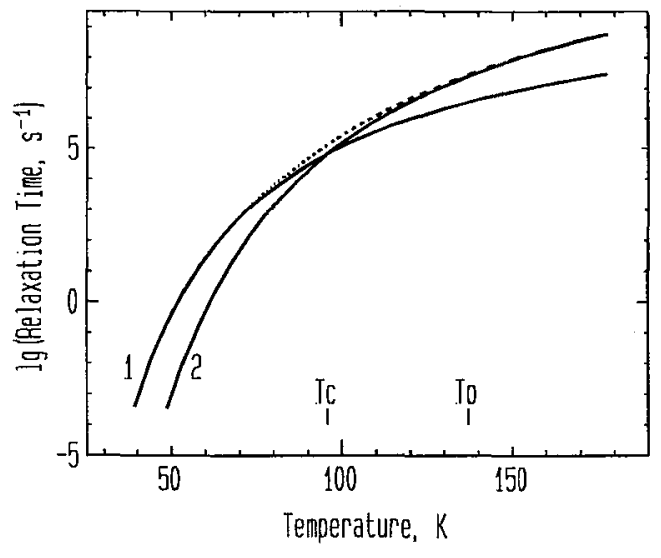

(a)

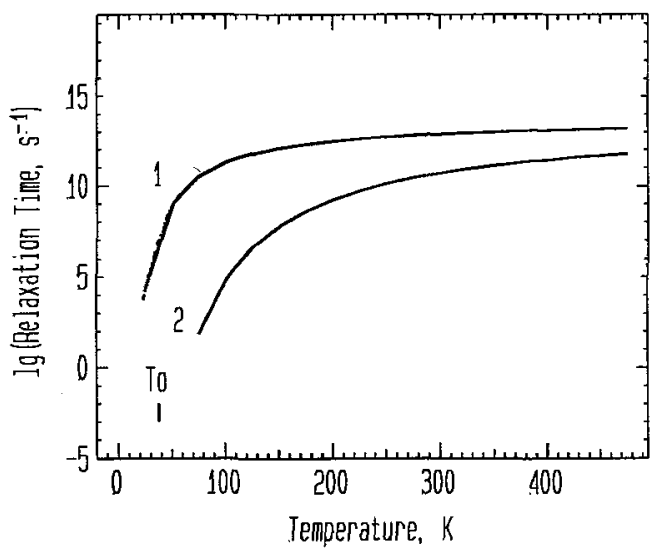

(b)

Figure 2. Calculated temperature dependencies of relaxation times of $\mathrm{H}^{-}$ion at $\mathrm{E}_{4}{ }_{4}$ defect center in $\alpha$-quartz(curves 1 TS relaxation times; curves 2 - RS relaxation times).

As it is mentioned above, a criterion for the applicability of the presented model is the comparison with the experimental data. There exist a peak at $26 \mathrm{~K}$ in dielectric losses in X-irradiated $\alpha$-quartz [4] at $f$ $=5 \mathrm{kHz}$. It is possible to compare the theoretical acoustic losses and the experimental dielectric ones because of the well known correspondence between the acoustic and dielectric losses in $\alpha$-quartz. The calculation of arbitrary acoustic losses due to $\mathrm{H}^{-}$ion relaxation (PJTE profile with $\Delta=0.063 \mathrm{eV}$ ) at $f=5 \mathrm{kHz}$ gives a peak at $T_{0}=27 \mathrm{~K}$. Regarding the transition probabilities, $\mathrm{H}$ ion is similar to TS (the lowest level tunneling transitions dominate the remaining level transitions practically for any temperature), while the distribution of the levels suggests that the losses have to be calculated as for the usual relaxation system. The agreement between the theoretical and experimental data (Fig. 3) allows to consider that the $\mathrm{H}$ ion is a TRS. 


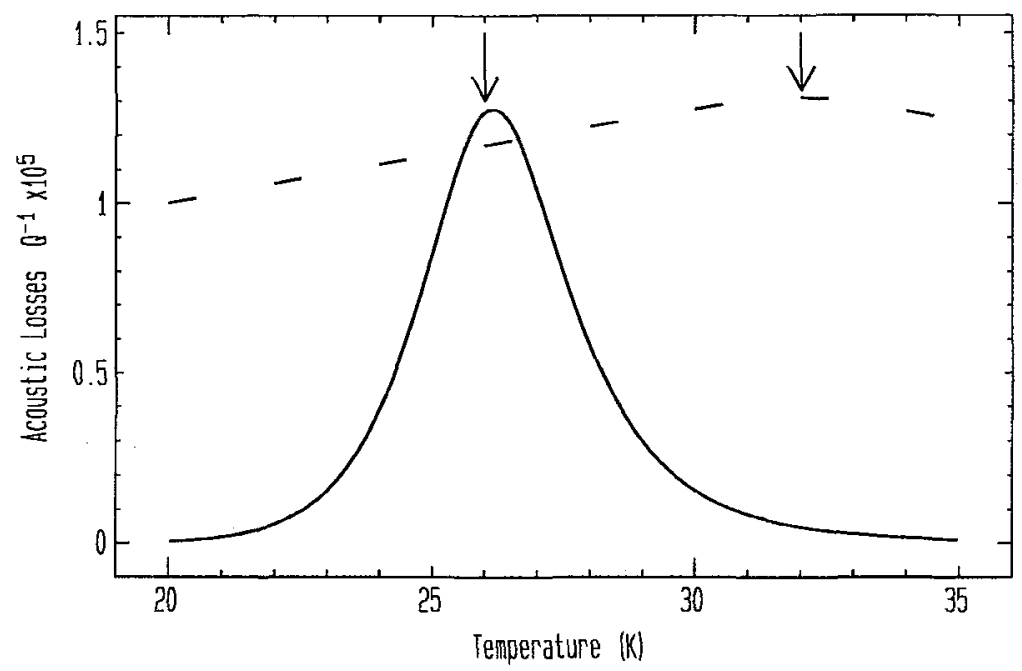

Figure 3. Temperature dependencies of the arbitrary acoustic losses due to $\mathrm{H}^{-}$ion at $\mathrm{E}_{4}{ }_{4}$ center in $\alpha$-quartz relaxation. Theoretical acoustic losses for the $\mathrm{H}^{-}$ion are compared with experimental dielectric losses (dashed line for fast-neutron irradiated quartz with maximum at $32 \mathrm{~K}$; the maximum for X-irradiated quartz appears at 26 $\mathrm{K}$. The maxima are marked with arrows) taken from [4].

\section{CONCLUSION}

Relaxation behavior of $\mathrm{H}^{-}$ion is considered on the base of new, precise fit of the cluster calculated data with PJT energy potential and numerical calculation of the eigenvalue spectrum. The improved method for the relaxation rates and losses calculation is applied. The temperature dependencies of the relaxation times and acoustic losses are calculated and compared with experimental data. The agreement between the theoretical and experimental results allows to consider that the $\mathrm{H}^{-}$ion is a TRS. $\mathrm{H}^{-}$ion is quite similar to TS because there exist one tunnel splitted energy level below the barrier and the tunneling transitions dominate the remaining ones practically for any temperature. On the other hand the thermal distribution of the levels occupation manifests that the losses have to be calculated as for the usual relaxation system. The presented theoretical results support the suggested method for description of the relaxation behaviour of $\mathrm{H}^{-}$ion at $\mathrm{E}_{4}^{\prime}$ defect centers and may be useful in forecasting the radiation response of $\alpha$-quartz.

\section{Acknowledgments}

The authors are grateful to "Drouzhba" Ltd. - Plovdiv, "SID" Ltd. - Sofia, and to the Organizing Committee of ICIFUAS-11, for the financial support.

\section{References}

[1] Georgiev M. and Manov A., Solid State Commun. 65 (1988) 6.

[2] Isoya J., Weil J. and Halliburton L., J. Chem. Phys. 74 (1981) 5436.

[3] Manov A. and Georgiev V., Acoustic Letters 14 (1991) 160.

[4] Stevels J. and Volger J., Philips Res. Repts. 17, 283 (1962).

[5] Weeks R.A. and Nelson C.M., J. Am. Ceram. Soc. 43 (1960) 399.

[6] Mihailov L., Kirtcheva M. and Manov A., Proc. Workshop on Quantum Systems in Chemistry and Physics, April 14-17, 1996, Pisa, Italy, to be published in Molecular Engineering..

[7] Christov S., Collision Theory and Statistical Theory of Chemical Reactions. Lecture Notes in Chemistry, Vol. 18, Berlin, Heidelberg, New York: Springer, 1980. 